\title{
Dopamine Triggers Heterosynaptic Plasticity
}

\author{
Masago Ishikawa, ${ }^{1 \star}$ Mami Otaka, ${ }^{1 \star}$ Yanhua H. Huang, ${ }^{2}$ Peter A. Neumann, ${ }^{1}$ Bradley D. Winters, ${ }^{3}$ Anthony A. Grace, ${ }^{1}$ \\ Oliver M. Schlüter, ${ }^{4}$ and Yan Dong ${ }^{1}$ \\ ${ }^{1}$ Department of Neuroscience and ${ }^{2}$ Department of Psychiatry, University of Pittsburgh, Pittsburgh, Pennsylvania 15260, ${ }^{3}$ Section of Neurobiology, \\ University of Texas at Austin, Austin, Texas 78712, and ${ }^{4}$ Molecular Neurobiology and Cluster of Excellence "Nanoscale Microscopy and Molecular \\ Physiology of the Brain”, European Neuroscience Institute, 37077 Göttingen, Germany
}

As a classic neuromodulator, dopamine has long been thought to modulate, rather than trigger, synaptic plasticity. In contrast, our present results demonstrate that within the parallel projections of dopaminergic and GABAergic terminals from the ventral tegmental area to the nucleus accumbens core (NAcCo), action-potential-activated release of dopamine heterosynaptically triggers LTD at GABAergic synapses, which is likely mediated by activating presynaptically located dopamine D1 class receptors and expressed by inhibiting presynaptic release of GABA. Moreover, this dopamine-mediated heterosynaptic LTD is abolished after withdrawal from cocaine exposure. These results suggest that action-potential-dependent dopamine release triggers very different cellular consequences from those induced by volume release or pharmacological manipulation. Activation of the ventral tegmental area to NAcCo projections is essential for emotional and motivational responses. This dopamine-mediated LTD allows a flexible output of NAcCo neurons, whereas disruption of this LTD may contribute to the rigid emotional and motivational state observed in addicts during cocaine withdrawal.

\section{Introduction}

Synaptic projections from the ventral tegmental area (VTA) to the nucleus accumbens (NAc) are essential for emotional and motivational responses (Wise, 1987). Within the VTA-to-NAc pathway are parallel dopaminergic and GABAergic projections, which are often concurrently activated upon the same emotional/ motivational stimuli (Steffensen et al., 1998; Carr and Sesack, 2000). This "intimate" anatomical and physiological partnership suggests that the VTA-to-NAc dopaminergic and GABAergic projections may exhibit unique interactions upon their coactivation during emotional and motivational responses.

Using optogenetic techniques combined with electrophysiological manipulations, we demonstrate here that activation of VTA-to-NAc dopaminergic projections triggers LTD at GABAergic synapses within this pathway. We further demonstrate that this LTD is triggered by heterosynaptic dopamine and is abolished after withdrawal from cocaine exposure. By pharmacological manipulations of dopamine receptors, extensive prior results firmly establish that dopamine is a modulator of plasticity. Our

\footnotetext{
Received Oct. 3, 2012; revised Jan. 27, 2013; accepted Feb. 22, 2013.

Author contributions: M.I., M.O., Y.H., A.A.G., O.M.S., and Y.D. designed research; M.I. and M.O. performed research; M.I., P.A.N., and B.D.W. analyzed data; M.I., M.O., Y.H., A.A.G., O.M.S., and Y.D. wrote the paper.

This work was supported by the National Institute on Drug Abuse-National Institutes of Health (A.A.G., Y.H., Y.D.), the Humboldt Foundation (Y.D.), the Hope Foundation for Depression Research (Y.D.), and the Deutsche Forschungsgemeinschaft through the Cluster of Excellence "Nanoscale Microscopy and Molecular Physiology of the Brain" and SCHL592/4 (0.M.S.). The European Neuroscience Institute-Göttingen is jointly funded by the Max-Planck Society and University Medicine Göttingen. We thank Dr. Sasha Nelson for providing the Gad2-cre mouse line.

*M.I. and M.O. contributed equally to this work.

The authors declare no competing financial interests.

Correspondence should be addressed to either of the following: Dr. Yan Dong, Department of Neuroscience, University of Pittsburgh, A215 Langley Hall, 5th and Ruskin Avenue, Pittsburgh, PA 15260, E-mail: yandong@pitt.edu; or Dr. Oliver M. Schlüter, European Neuroscience Institute, Grisebachstr. 5, 37077 Göttingen, Germany, E-mail: oschlue@gwdg.de.

DOI:10.1523/JNEUROSCI.4694-12.2013

Copyright $\odot 2013$ the authors $\quad 0270-6474 / 13 / 336759-07 \$ 15.00 / 0$
}

present results indicate that action-potential-driven dopamine release is also a trigger of plasticity. This form of LTD relieves NAc neurons from inhibition upon receipt of phasic input from dopamine neurons that has been shown to be triggered by reward or reward-associated cues (Fiorillo et al., 2003). Disruption of this LTD may contribute to the rigidity in reward-associated responses that occur during cocaine withdrawal.

\section{Materials and Methods}

Viral vectors. Channelrhodopsin-2 (ChR2) fused to Venus (AAVChR2Y; Addgene plasmid 20071) or mCherry (AAV-flexed-ChR2R; Addgene plasmid 18916) were expressed from an adeno-associated viral vector 2 (AAV2) with AAV2 internal repeats (Atasoy et al., 2008; Petreanu et al., 2009; Suska et al., 2013). AAV-flexed ChR2R expression was induced specifically in Cre-recombinase-expressing neurons, in which the inverted expression cassette was flipped (Atasoy et al., 2008). In this study, flexed virus was used in Gad-cre or TH-cre mouse lines.

Animal use, stereotaxic injections, and cocaine administration. Male Sprague-Dawley rats (Simonsen), the Gad2-IRES-Cre mouse line (Gad2 ${ }^{\mathrm{tm} 2(\mathrm{cre}) \mathrm{Zjh}}$; The Jackson Laboratory), and the TH-IRES-Cre mouse line (B6.129X1-Th ${ }^{\mathrm{tm} 1(\mathrm{cre}) \mathrm{Te}} / \mathrm{Kieg}$; European Mouse Mutant Archive; Lindeberg et al., 2004) were used. Intra-VTA viral injections were performed when animals were at the age of postnatal 26-34 d. Virus was delivered stereotaxically in animals anesthetized with a mixture of ketamine/xylazine (50/6 mg/kg; Huang et al., 2011). A 28 -gauge needle was used to bilaterally inject $1 \mu \mathrm{l}(0.2 \mu \mathrm{l} / \mathrm{min})$ of the virus solution into the VTA (rat: AP/ML/DV: $-5.00 / \pm 0.90 /-7.65 \mathrm{~mm}$; mouse: $-3.44 / \pm 0.48 /$ $-4.4)$. Experiments were performed $\sim 3$ weeks after viral injection. After recovery, rats received intraperitoneal injections of cocaine $(15 \mathrm{mg} / \mathrm{kg} / \mathrm{d}$ in saline for $5 \mathrm{~d})$ or saline $(0.1 \mathrm{ml} / 100 \mathrm{~g})$ in a novel environment (Ishikawa et al., 2009; Mu et al., 2010; Brown et al., 2011). Rats were placed back in the home cage for withdrawal.

NAc slice preparations and electrophysiology. As described previously (Ishikawa et al., 2009; Mu et al., 2010; Huang et al., 2011), the animals were decapitated after being given isoflurane anesthesia. The brain was sliced in $4^{\circ} \mathrm{C}$ cutting solution containing the following (in $\mathrm{mm}$ ): 135 

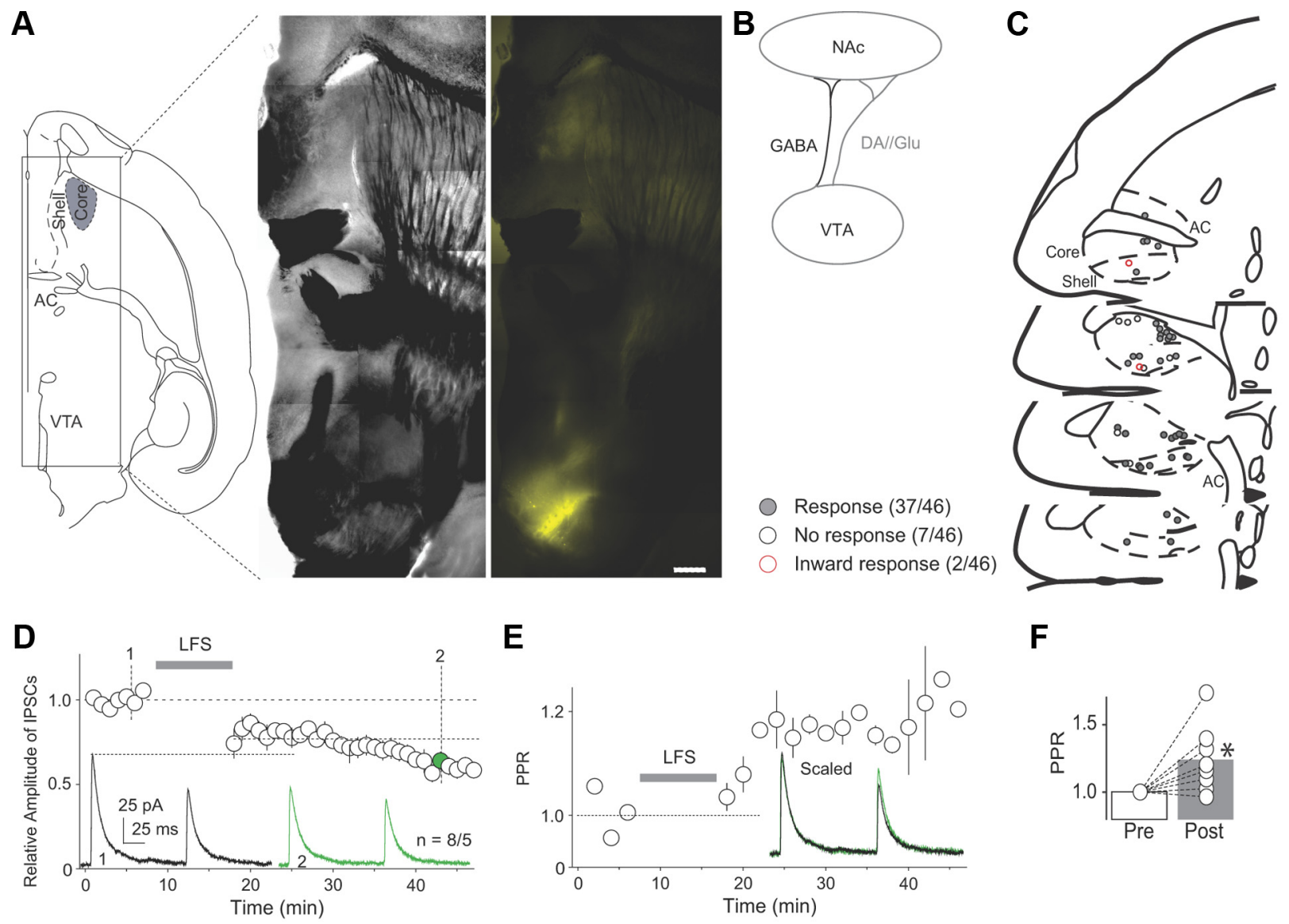

$\mathbf{F}$
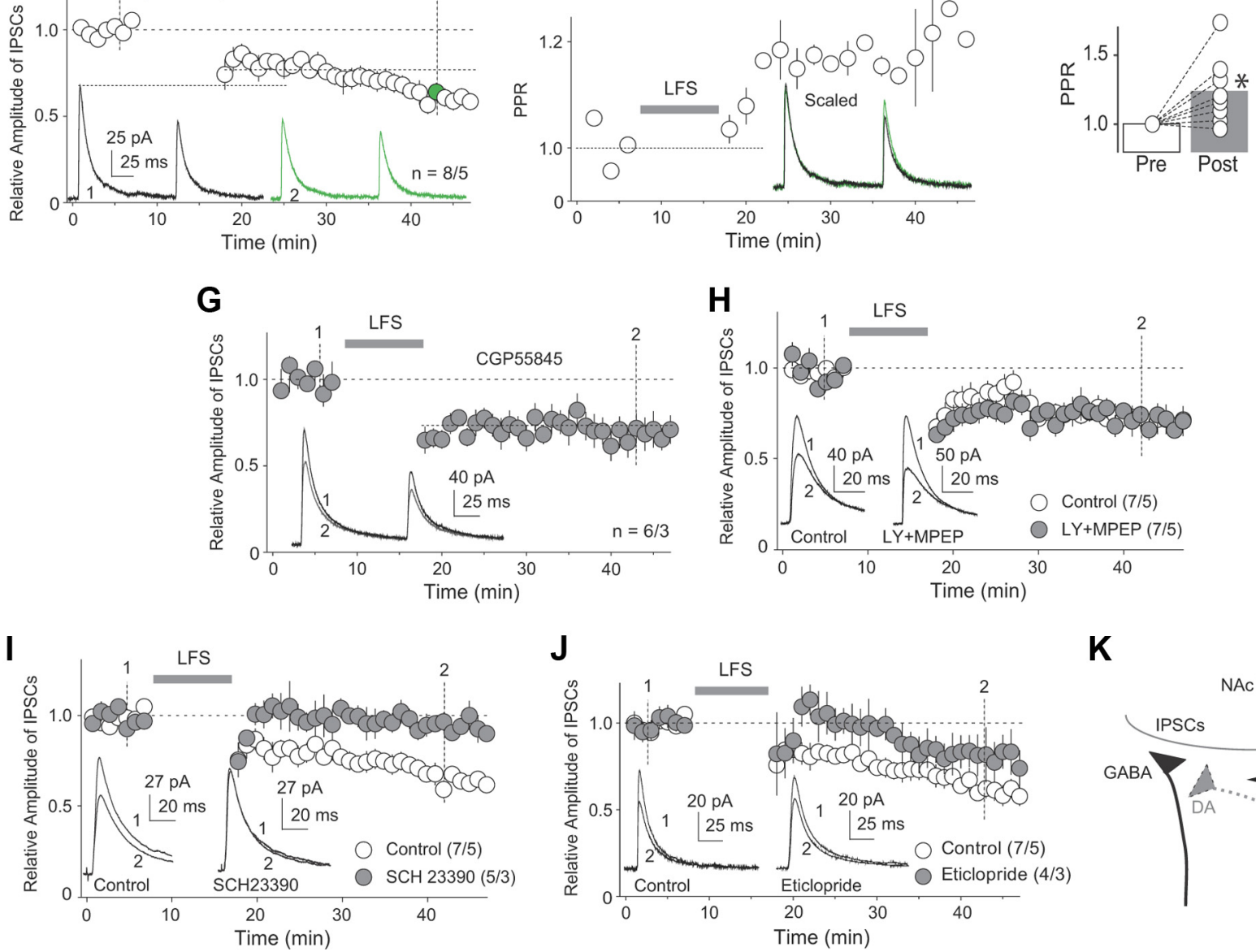

K

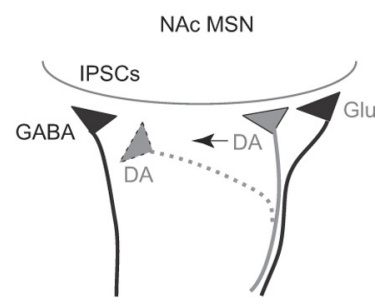

Figure 1. LTD at VTA-to-NACCO GABAergic synapses. $A$, Diagrams and images showing that intra-VTA injection of ChR2-expressing virus resulted in expression of ChR2 in fibers within the NAcCore in a horizontal brain slice. Scale bar, $500 \mu \mathrm{m}$. B, Diagram showing that in the rat preparation, VTA-to-NAcCo projections include both GABAergic and dopaminergic/glutamatergic fibers. C, Diagrams showing locations of recorded neurons exhibiting IPSCs (responses), no responses, and inward synaptic currents upon optical stimulations. D, Summarized results showing LTD induced by an LFS protocol at VTA-to-NAcCo GABAergic synapses. Inset: Example of IPSCs before and after LTD induction. Numbers (1 and 2) indicate the time points when the example IPSCs were taken. $E$, Time course of the PPR of IPSCs in D. Inset: Scaled IPSCs of the examples shown in D.F, Summarized results showing that this LTD was accompanied by an increase in the PPR. G, Summarized results showing that this LTD was not prevented in the presence of the $G A B A_{B}$-receptor-selective antagonist CGP55845. $\boldsymbol{H}$, Summarized results showing that this LTD was not prevented in the presence of $m G l u R 1$ - and mGluR5-selective antagonists LY367385 and MPEP.I, Summarized results showing that this LTD was prevented by the dopamine D1R-selective antagonist SCH23390.J, Summarized results showing that this LTD was not prevented by the dopamine D2R-selective antagonist eticlopride. $\boldsymbol{K}$, Diagram showing that the parallel dopaminergic terminals may provide heterosynaptic modulation of VTA-to-NAcCo GABAergic transmission. ${ }^{*} p<0.05$. 
$N$-methyl-D-glucamine, $1 \mathrm{KCl}, 1.2 \mathrm{KH}_{2} \mathrm{PO}_{4}, 0.5 \mathrm{CaCl}_{2}, 1.5 \mathrm{MgCl}_{2}, 20$ choline- $\mathrm{HCO}_{3}$, and 10 glucose, saturated with $95 \% \mathrm{O}_{2} / 5 \% \mathrm{CO}_{2}$, pH adjusted to 7.4. Slices were incubated in artificial CSF containing the following (in mM): $119 \mathrm{NaCl}, 2.5 \mathrm{KCl}, 2.5 \mathrm{CaCl}_{2}, 1.3 \mathrm{MgCl}_{2}, 1 \mathrm{NaH}_{2} \mathrm{PO}_{4}$, $26.2 \mathrm{NaHCO}_{3}$, and 11 glucose, saturated with $95 \% \mathrm{O}_{2} / 5 \% \mathrm{CO}_{2}$ at $37^{\circ} \mathrm{C}$ for $30 \mathrm{~min}$ and then at room temperature before experimentation.

During recordings, slices were superfused with artificial CSF at 31$33^{\circ} \mathrm{C}$. Recordings were made with electrodes (3-5 M $\Omega$ ) filled with (in $\mathrm{mm}$ ): $140 \mathrm{CsCH}_{3} \mathrm{O}_{3} \mathrm{~S}, 5$ TEA-Cl, 0.4 EGTA, 20 HEPES, 2.5 Mg-ATP, 0.25 Na-GTP, and 1 QX-314, pH 7.3.

To evoke EPSCs/IPSCs, axons expressing ChR2 were stimulated by $473 \mathrm{~nm}$ laser (IkeCool) coupled to a $62.5 \mu \mathrm{m}$ optic fiber. The optical stimulation was given with $0.05-1 \mathrm{~ms}$ duration at $0.1 \mathrm{~Hz}$. EPSCs were recorded at $-70 \mathrm{mV}$ and IPSCs at $+10 \mathrm{mV}$. To induce presynaptically expressed LTD of IPSCs, a modest protocol $(5 \mathrm{~Hz} \times 10 \mathrm{~min})$ was shown to be sufficient (Chiu et al., 2010). In our experiments, an even more modest protocol $(2 \mathrm{~Hz} \times 10 \mathrm{~min})$ could reliably induce LTD at VTA-toNAcCo inhibitory synapses.

All chemicals were purchased from Sigma-Aldrich or Tocris Bioscience. For all recordings, series resistance was 8-14 M $\Omega$ and was left uncompensated. Series resistance was monitored continuously, and a change $>20 \%$ was not accepted for data analysis. Synaptic currents were recorded with a MultiClamp 700B amplifier (Molecular Devices) filtered at $2.6-3 \mathrm{kHz}$ and digitized at $20 \mathrm{kHz}$.

All procedures were performed by strictly following the standard procedures approved by the animal care and use committees of the listed institutions.

Data analysis and statistics. All results are shown as mean \pm SEM. Statistical significance was assessed using either one- or two-factor ANOVA with Bonferroni posttests or two-tailed $t$ test. One to four cells were recorded from each animal. The total numbers of cells/animals are presented as "n/m." Cell-based statistics were performed for all results.

\section{Results}

To record IPSCs from the VTA to NAc core (NAcCo) medium spiny neurons (MSNs), we used viral-mediated gene transfer with AAV to express Venus-tagged ChR2 (ChR2Y) in the VTA in vivo. Three weeks later, we obtained horizontal brain slices within which the VTA exhibited yellow fluorescent signals, indicating the expression of ChR2 (Fig. 1A). Within the same slices, ChR2Ypositive neural fibers were observed projecting from the VTA to the forebrain including the NAc (Fig. 1A). By laser-lightmediated activation of ChR2 $(0.05-1 \mathrm{~ms}, \lambda=473 \mathrm{~nm})$, VTA-toNAcCo GABAergic and dopaminergic/glutamatergic synaptic transmissions were activated simultaneously (Fig. 1B). GABAergic transmission was recorded at $+10 \mathrm{mV}$, near the reversal potential of glutamatergic transmission. IPSCs were detected in most (37/46) recorded NAcCo neurons (Fig. 1C); neurons with no response or slow inward current were excluded from subsequent experiments.

We focused on LTD at VTA-to-NAcCo GABAergic synapses, a form of synaptic plasticity that may relieve NAcCo MSNs from basal inhibition of their output. We optimized a low-frequency stimulation (LFS; $2 \mathrm{~Hz} \times 10 \mathrm{~min}$ ) induction protocol, delivery of which via optical stimulation induced a reliable LTD at VTA-toNAcCo inhibitory synapses ( $p<0.01, t$ test; Fig. $1 D)$. Two prominent features of this LTD were: (1) it continued developing during the $50 \mathrm{~min}$ recording, a property similar to LTD of IPSCs in other brain regions (Morishita and Sastry, 1996; Heifets et al., 2008; Chiu et al., 2010) and (2) it was accompanied by an increase in the paired-pulse ratio (PPR), suggesting a presynaptic site of expression $(p<0.05$, paired $t$ test; Fig. $1 D-F)$.

Under this experimental setup, both GABAergic and dopaminergic/glutamatergic signaling was activated during LTD induction, of which the $\mathrm{GABA}_{\mathrm{B}^{-}}$(Chalifoux and Carter, 2011) and dopamine/glutamate-signaling pathways (Castillo et al., 2011)
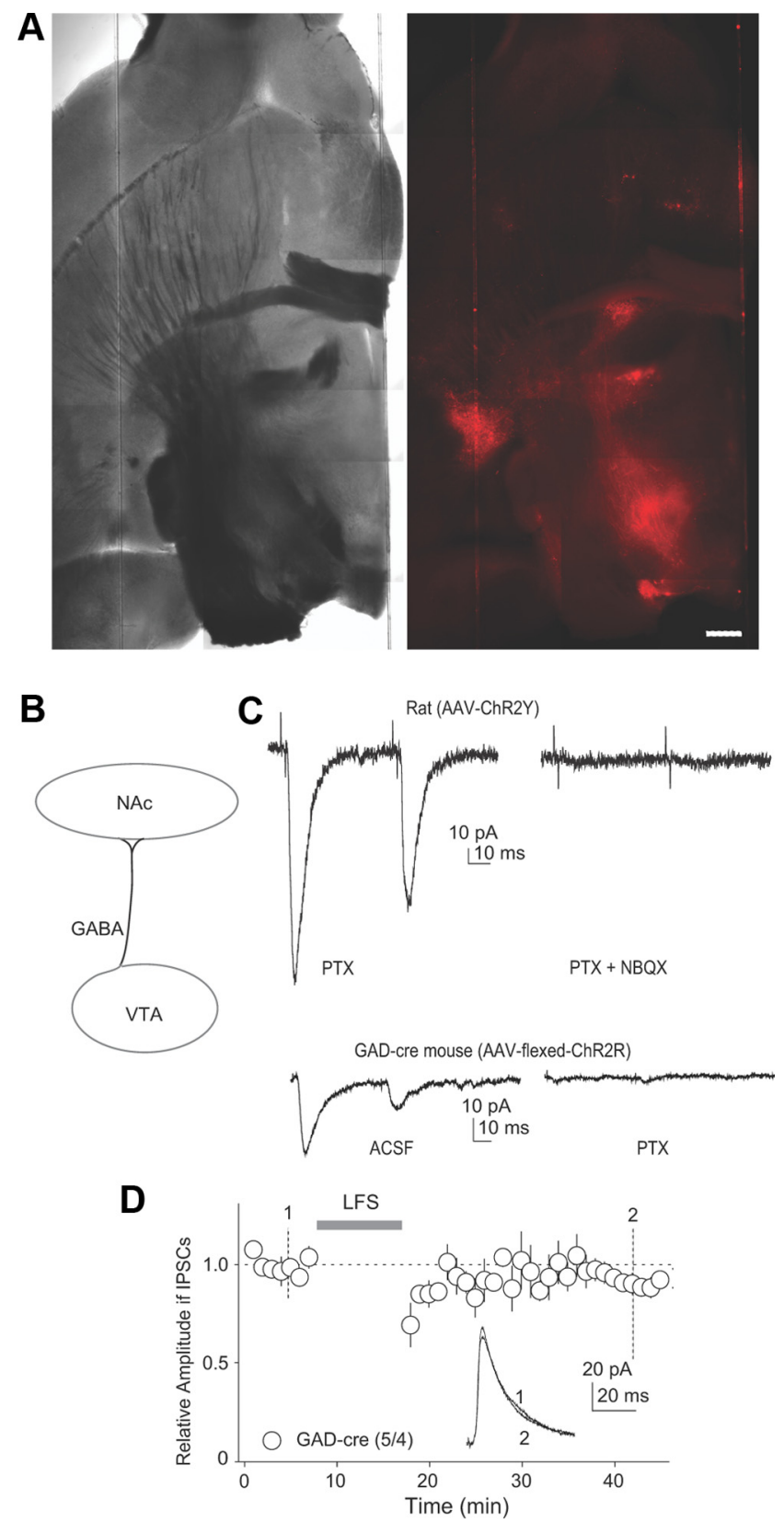

Figure 2. LTD at VTA-to-NACCo inhibitory synapses could not be induced by activation of these synapses alone. $A$, Images showing that intra-VTA injection of flexed ChR2R resulted in expression of ChR2R (red fluorescent signals) in fibers within the NAcCo in a horizontal brain slice from a GAD-cre mouse. Scale bar, $500 \mu \mathrm{m}$. B, Diagram showing that with this optogenetic approach, optical stimulation activated VTA-to-NACCo GABAergic transmission selectively. $C$, Example traces showing that in brain slices prepared from rats (top) in which ChR2-AAV was injected, in addition to IPSCs, optical stimulation often elicited EPSCs (recorded at $-70 \mathrm{mV}$, sensitive to NBQX), suggesting an infection of VTA-to-NAcCo dopaminergic/glutamatergic projection. In brain slices prepared from GAD-cre mice in which ChR2-flexed AAV was injected (bottom), optical stimulation rarely elicited EPSCS, suggesting that only the GABAergic projection was infected. $\boldsymbol{D}$, Summarized results showing that when the GABAergic VTA-to-NACCO projection was stimulated selectively (flexed ChR2R in GAD-cre mice), LFS failed to induce LTD. Inset: Example of IPSCs before and after LFS stimulation.

have been shown to regulate inhibitory synaptic transmission presynaptically. Our subsequent results did not suggest a predominant role of $\mathrm{GABA}_{\mathrm{B}}$ signaling; inhibition of $\mathrm{GABA}_{\mathrm{B}}$ receptors by superfusion of $\mathrm{GABA}_{\mathrm{B}}$-receptor-selective antagonist CGP55845 $(10 \mu \mathrm{M})$ did not prevent LFS-induced LTD $\left(F_{(3,24)}=20.79, p<0.01\right.$, onefactor ANOVA; $p<0.01$, CGP55845 prestimulation vs CGP55845 


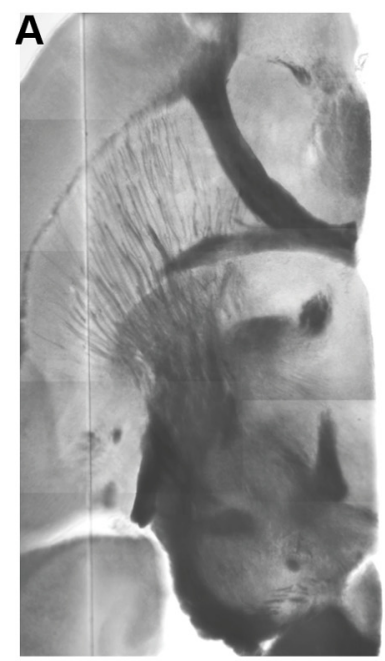

D

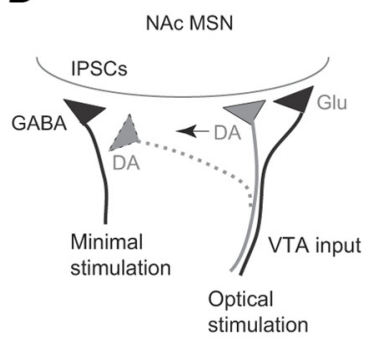

$\mathbf{E}$

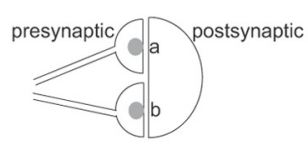

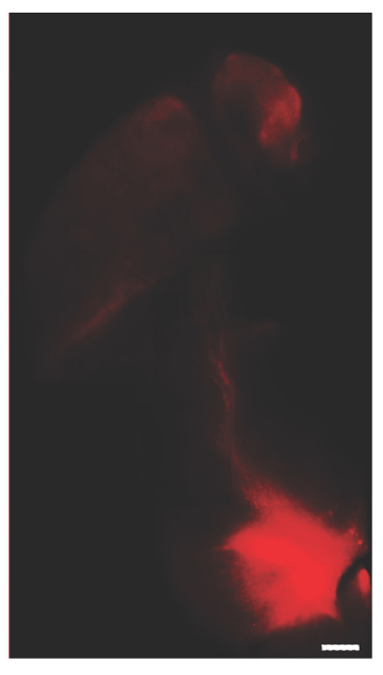

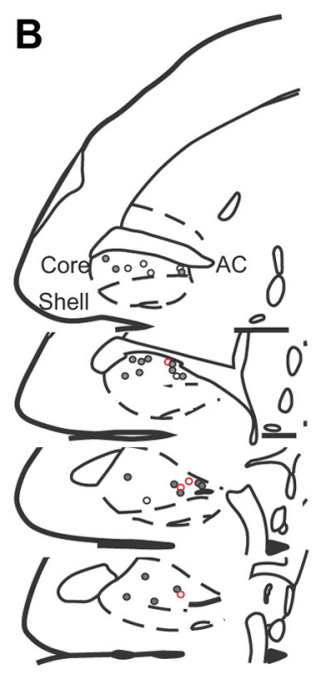

No response (10/33)

Rundown (19/33)

Stable response $(4 / 33)$

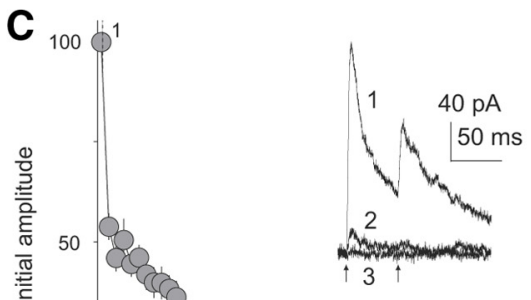

$\mathbf{F}$
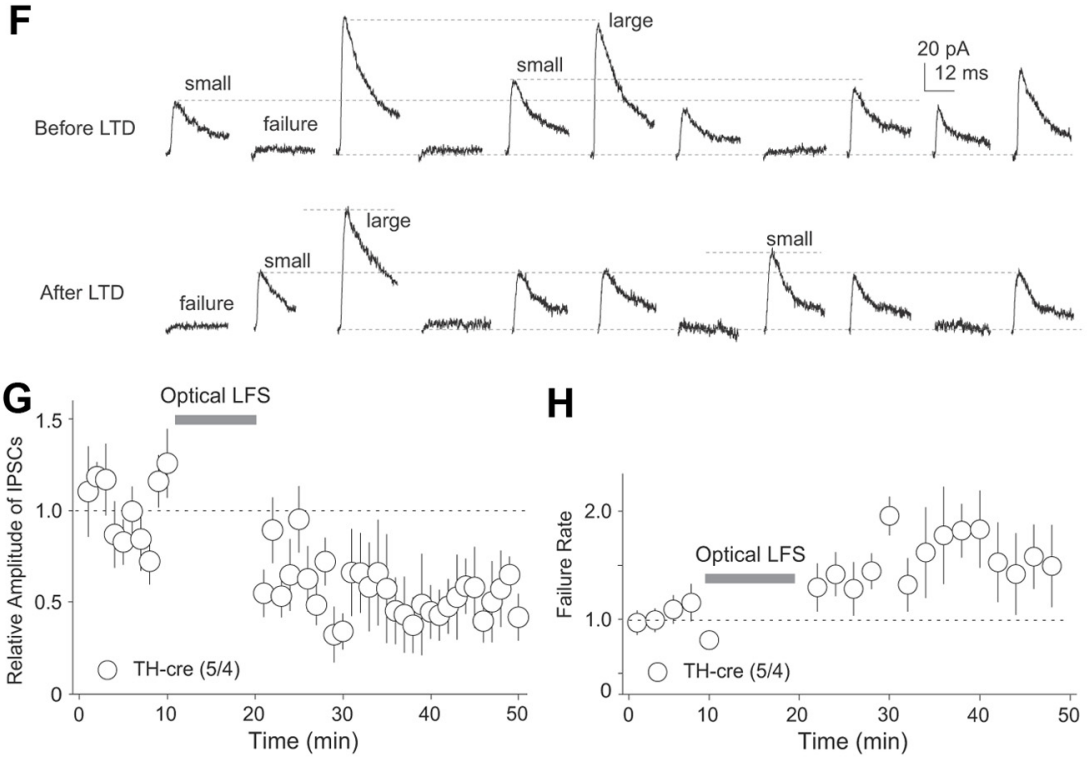

H
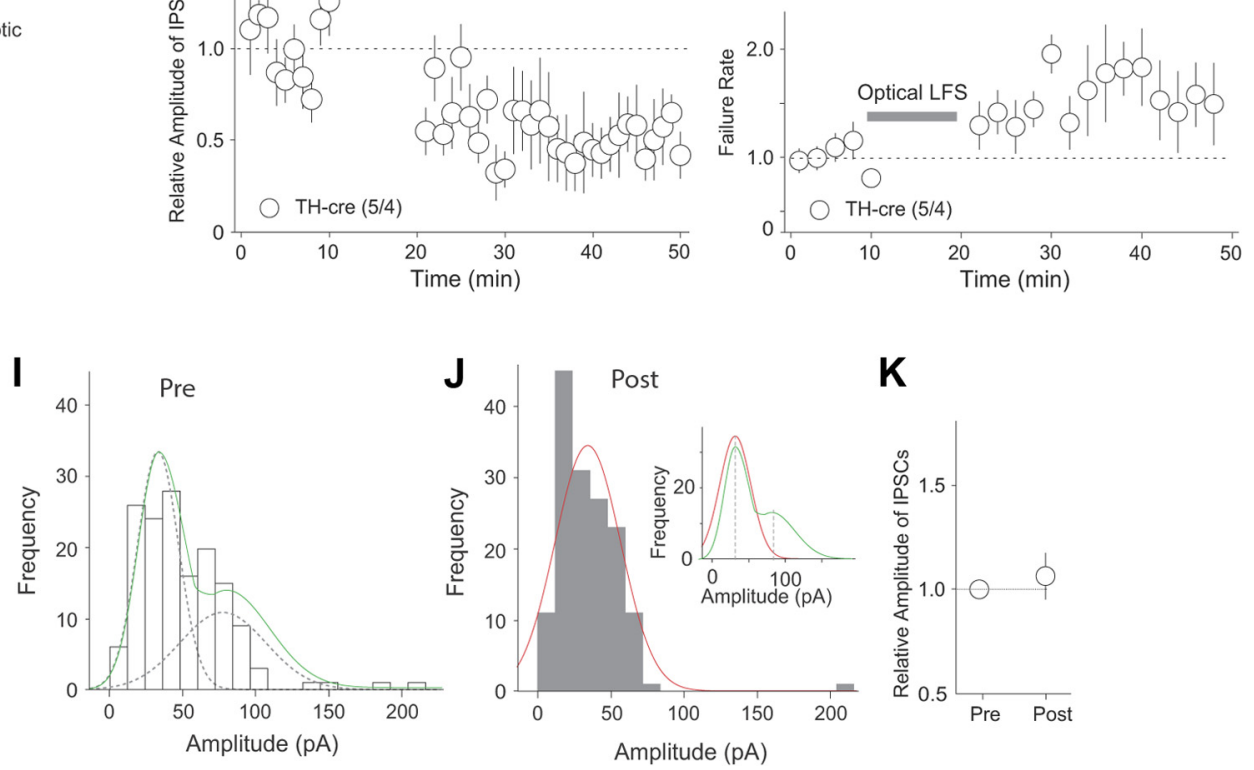

Figure 3. LTD by heterosynaptic dopamine. $A$, Images showing that intra-VTAinjection offlexed ChR2R resulted in expression of ChR2R (red fluorescent signals) in fibers within the NAcCo in a horizontal brain slice from a TH-cre mouse. Scale bar, $500 \mu \mathrm{m}$. B, Diagram showing the locations of recorded NAcCo neurons that exhibited no synaptic responses, IPSCs with significant rundown, and stable IPSCs in a subset of experiments. C, Summarized results showing that IPSCs from VTA-to-NAcCo dopaminergic projections declined rapidly during recording. Stimulation frequency, $0.1 \mathrm{~Hz}$. D, Diagram showing the experimental setup for minimal stimulation assay in which GABAergic synaptic transmission was evoked by electrical stimulation in a minimal stimulation protocol and activation of dopamine signaling was achieved by optical stimulation. E, Diagram showing an example of the minimal stimulation setup in which two synapses are included by the stimulation. Three types of postsynaptic responses can be generated: (1) failures, when both synapses fail to release neurotransmitter; (2) small responses, when one of the two synapses is activated successfully; and (3) large responses, when both synapses are activated simultaneously. The incidences offailures, small responses, or large responses are determined by the presynaptic release probability. $F$, Example of IPSCs evoked by the minimal stimulation protocol in NAcCo neurons before and after the induction of LTD. G, Summarized results showing that LTD was induced by the optically applied LFS (only dopaminergic fibers were activated by this LFS). All responses including failures were included in this summary. $\boldsymbol{H}$, Summarized results showing that the LTD was accompanied by an increase in the failure rate. I, J, Summarized results showing that the LTD induction did not change the amplitude of small responses, but reduced the incidence of large responses. The amplitudes of IPS(S evoked by the minimal stimulation protocol were well fit by two-Gaussian distributions before LTD induction (I), presumably corresponding to the small and large responses, and one Gaussian distribution after LTD $(J)$. Inset in $J$ shows that the amplitude of small responses did not change, whereas the incidence of large responses was reduced. $\boldsymbol{K}$, Summarized results showing that the amplitude of small responses was not altered by LTD induction. 
A
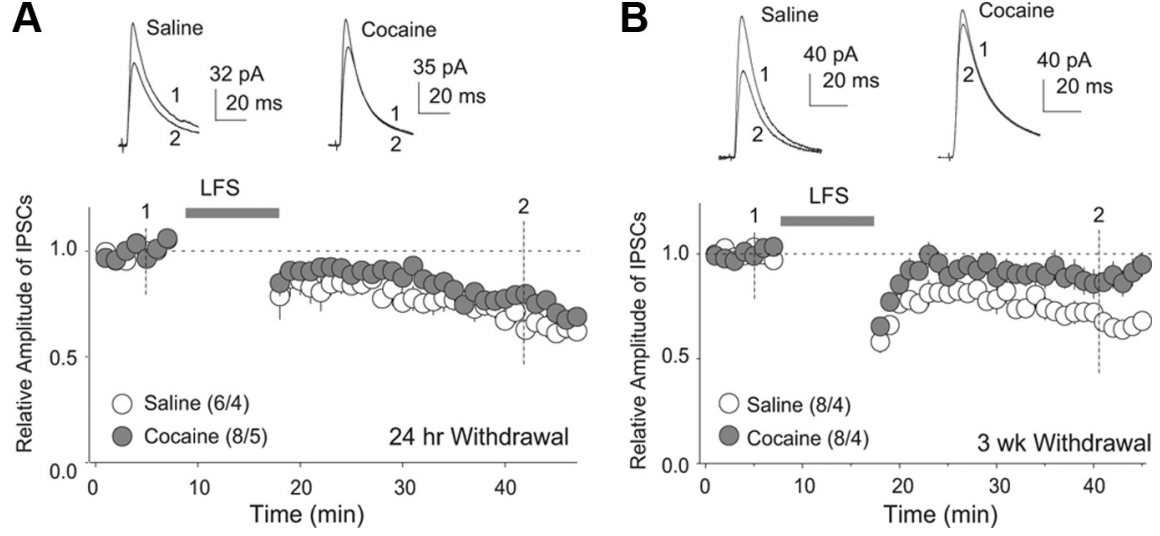

Figure 4. LTD at VTA-to-NAcCo inhibitory synapses is disrupted after cocaine withdrawal. $\boldsymbol{A}$, Summarized results showing that LTD at VTA-to-NACCo inhibitory synapses was slightly attenuated after $1 \mathrm{~d}$ withdrawal from repeated intraperitoneal injections of cocaine. Insets show examples of IPSCS at the time points indicated by the numbers. $B$, Summarized results showing that LTD at VTA-to-NAcCo inhibitory synapses was significantly attenuated after a 3-week withdrawal from repeated intraperitoneal injections of cocaine. Insets show examples of IPSCs at the time points indicated by the numbers.

poststimulation; $p=1.0$, control poststimulation vs CGP55845 poststimulation, Bonferroni posttests; Fig. $1 G)$. However, inhibition of $\mathrm{GABA}_{\mathrm{B}}$ receptors abolished the continuously developing feature of this LTD (Fig. $1 G$ ). Upon activation of dopaminergic terminals, it is possible that glutamate is co-released to activate postsynaptic group I metabotropic glutamate receptors (mGluRs). This may induce a release of endocannabinoids, triggering presynaptically expressed LTD (Castillo et al., 2011). However, coinhibition of mGluR 1 and 5 by superfusion of their selective antagonists LY367385 (100 $\mu \mathrm{M})$ and $\operatorname{MPEP}(10 \mu \mathrm{M})$ did not prevent this $\operatorname{LTD}\left(F_{(3,24)}=17.4, p<\right.$ 0.01 , one-factor ANOVA; $p<0.01$, prestimulation vs poststimulation in antagonists; $p=1.0$, control poststimulation vs antagonists poststimulation, Bonferroni posttests; Fig. $1 H$ ). In contrast to $\mathrm{GABA}_{\mathrm{B}}$ and mGluRs, inhibition of dopamine D1-class receptors (D1Rs) by SCH23390 $(1 \mu \mathrm{M})$ prevented the induction of LTD $\left(F_{(3,20)}=30.06, p<0.01\right.$, one-factor ANOVA; $p=1.0$, SCH 23390 prestimulation vs SCH23390 poststimulation; $p<0.01$, control poststimulation vs SCH23390 poststimulation, Bonferroni posttests; Fig. 1I). Furthermore, inhibition of dopamine D2-class receptors (D2Rs) by eticlopride ( $3 \mu \mathrm{M})$ did not completely prevent LTD $\left(F_{(3,18)}=12.56, p<0.01\right.$, one-factor ANOVA; $p=0.20$, control poststimulation vs eticlopride poststimulation; $p=0.28$, eticlopride prestimulation vs eticlopride poststimulation, Bonferroni posttests; Fig. $1 J)$.

These results suggest that induction of this LTD required dopamine signaling, which was likely a novel form of heterosynaptic plasticity involving the parallel VTA-to-NAcCo dopaminergic terminals; the heterosynatpic nature of this plasticity is likely mediated by nearby dopaminergic terminals via local heterosynaptic transmission or "spillover" of dopamine from distal dopaminergic terminals (Fig. $1 K$ ). We next tested this possibility by studying VTA-to-NAcCo GABAergic and dopaminergic/glutamatergic inputs using optogenetic tools.

To isolate GABAergic input, we used GAD-cre mice (Taniguchi et al., 2011), in which we injected an inducible AAV-ChR2R (flexed-ChR2R) into the VTA. Therefore, only GABAergic neurons, but not dopaminergic/glutamatergic neurons, expressed ChR2R and projected ChR2R-expressing fibers to the NAcCo (Fig. 2A,B). Although it has been reported that very few VTA GABAergic neurons project to NAc MSNs (Brown et al., 2012), stable IPSCs were detected in the present study. Given that our recordings were focused on the caudal NAcCo (Fig. 1C), the dis- crepant results may reflect that NAcCo neurons within different subregions possess different synaptic connections. Nonetheless, the efficacy was assessed electrophysiologically. Briefly, because dopamine is co-released with glutamate at these terminals (Sulzer et al., 1998; Lavin et al., 2005; Nair-Roberts et al., 2008; Stuber et al., 2010), activation of dopaminergic fibers can be detected operationally by the presence of EPSCs (Fig. 2C). In rat brain slices with intra-VTA injection of regular AAVChR2Y, laser stimulation elicited both IPSCs (picrotoxin sensitive) and EPSCs (NBQX sensitive) in most of the recorded neurons (35/36). In contrast, in GAD-cre mice receiving AAV-flexed ChR2R, NBQXsensitive currents were rarely observed (4/ 30; Fig. 2C), suggesting that the VTA GABAergic projection was isolated successfully. Using this approach, we found that laser-mediated LFS did not induce LTD at VTA-to-NAcCo inhibitory synapses ( $p=0.09$, paired $t$ test; Fig. $2 D)$, indicating that activation of GABAergic transmission alone did not induce LTD and that the LTD is likely heterosynaptic in nature.

To determine whether heterosynaptic dopamine signaling was the key, we combined optogenetic techniques with the minimal stimulation assay. We used TH-cre mice (Lindeberg et al., 2004) that received intra-VTA injection of AAV-flexed ChR2R. Therefore, only dopaminergic neurons expressed ChR2R and projected ChR2R-expressing fibers to the NAcCo (Fig. $3 A$ ). The dopaminergic/glutamatergic specificity of this approach was confirmed by the consistent appearance of EPSCs in NAcCo neurons upon optical stimulation (11/12). In addition, as described previously (Tritsch et al., 2012), activation of VTA-to-NAcCo dopaminergic/glutamatergic terminals also elicited picrotoxinsensitive $(100 \mu \mathrm{M})$ IPSCs in more than half (23/33) of recorded NAcCo neurons (Fig. 3B). However, IPSCs evoked from VTAto-NAcCo dopaminergic projection exhibited a significant rundown by $\sim 50 \%$ within the first minute and declined by $\sim 90 \%$ within 5 min (Fig. 3C). This decaying kinetics is consistent with the notion that GABA released from the VTA-to-NAcCo dopaminergic projection is "packaged" together with dopamine and released from the same vesicles (Tritsch et al., 2012). Because the baseline of our LTD experiments was typically established after 10 min of recording, it is not likely that GABA release from the dopaminergic projection contributed significantly to either the baseline IPSCs or IPSCs after LTD shown in Figure 1. Furthermore, these results do not support a role for GABAergic signaling in the induction of this LTD (Fig. 1G, Fig. 2D). Therefore, we did not focus on this atypical transmission in the subsequent assays.

Within the NAc slices containing ChR2R-expressing dopaminergic fibers, we established a minimal stimulation procedure (Fig. 3D). Through an electrical stimulation electrode, we applied weak stimulations such that only a small number of inhibitory synapses were included (recorded at around the reversal potential of EPSCs). Although this approach allowed for the examination of individual inhibitory synapses, these synapses did not necessarily originate from the VTA. In the example shown in Figure $3 E$, two functional synapses were included, which should produce three types of postsynaptic responses throughout the trial: (1) failures, when both synapses fail to release neurotransmitters; (2) small responses, when one of the two synapses is successfully 
activated (depending on the actual properties and locations of these two putative synapses, small responses may not be identical); and (3) large responses, when both synapses are activated simultaneously. The incidence of failures, small responses, or large responses is determined by the presynaptic release probability. This technique offers two detectable parameters with which to assess the presynaptic or postsynaptic expression mechanisms of LTD: the failure rate, for which a decreased release probability would be accompanied by an increased rate of failures and a decreased rate of large responses, and the amplitude of small responses, which is likely mediated by a single unitary response.

We recorded IPSCs from NAcCo neurons evoked by minimal stimulation and then applied LFS to the VTA-to-NAcCo dopaminergic/glutamatergic input using optical stimulations. Our results show that the overall amplitude of IPSCs (including responses and failures) was decreased in an LTD-like manner $(p=0.01$, paired $t$ test; Fig. $3 F, G)$. This LTD was accompanied by an increased failure rate, suggesting a decrease in presynaptic release probability ( $p=0.04$, paired $t$ test; Fig. $3 H$ ). Furthermore, when the Gaussian distributions of all responses (from all recorded cells) were plotted before and after the LTD induction, it became apparent that the LTD procedure reduced the incidence of large responses without affecting the amplitude of small responses $(p=0.59$, paired $t$ test; Fig. $3 I-K)$. These results suggest that activation of heterosynaptic dopamine signaling is sufficient to induce presynaptically expressed LTD identified at VTA-to-NAcCo inhibitory synapses (Fig. 1).

By compromising the ability of synapses to undergo plastic changes, exposure to cocaine or other drugs of abuse could potentially reduce the flexibility of the brain during its response to emotional/motivational stimuli (Kasanetz et al., 2010). Consistent with this, we observed in rats that the LTD at VTA-toNAcCo inhibitory synapses was minimally reduced after $1 \mathrm{~d}$ withdrawal after repeated exposure to cocaine $\left(F_{(3,24)}=36.24\right.$, $p<0.01$, one-factor ANOVA; $p=0.39$, saline poststimulation vs cocaine poststimulation; $p<0.001$, cocaine prestimulation vs cocaine poststimulation, Bonferroni posttests; Fig. 4A), but largely abolished when the withdrawal time extended to 3 weeks $\left(F_{(3,28)}=21.38, p<0.01\right.$, one-factor ANOVA; $p<0.01$, saline poststimulation vs cocaine poststimulation; $p=0.13$, cocaine prestimulation vs cocaine poststimulation, Bonferroni posttests; Fig. $4 B$ ). Note that the pattern of LTD was slightly altered in an age-dependent manner.

\section{Discussion}

GABAergic neurons constitute approximately one-third of the neuronal population of the VTA, receive synaptic projections from the same brain regions that innervate dopaminergic neurons, project together with dopaminergic neurons to the same brain regions, and are activated simultaneously with dopaminergic neurons upon reward stimulation (Steffensen et al., 1998; Carr and Sesack, 2000; Nair-Roberts et al., 2008). Despite such an "intimate" anatomic setup, little is known regarding how VTA dopamine and GABA transmissions interact. Our present results demonstrate a form of LTD at the VTA-to-NAcCo GABAergic projection that is triggered by the parallel dopaminergic signaling. This heterosynaptic LTD was disrupted after withdrawal from cocaine administration. These findings may provide insight into understanding the mechanisms of reward- and cocaineinduced cellular and circuitry responses, as described below.

First, although experience-dependent plasticity at inhibitory synapses has been observed in many brain regions (Castillo et al.,
2011), a strikingly different feature of the currently identified LTD is that it relies on heterosynaptic dopaminergic signaling. It has long been thought that dopamine acts as a neuromodulator but does not induce long-term synaptic plasticity directly (Calabresi et al., 2007). Our present study represents one of the first demonstrations that heterosynaptic dopamine signaling can trigger synaptic plasticity independently, unveiling another biological function of dopamine. This feature is particularly important for the physiological role of VTA-to-NAcCo GABAergic transmission. VTA GABAergic neurons spike continuously in vivo (Steffensen et al., 1998). This constant firing is translated into stable background inhibitory control of NAc neurons only if the firing does not induce adaptive changes, which based on our results is the case (Fig. 2). Furthermore, upon exposure to incentive stimuli, VTA dopaminergic neurons increase firing and the resulting heterosynaptic dopamine signaling may induce incentive-contingent LTD of VTA-to-NAcCo inhibitory transmission. Indeed, based on the results in the minimal stimulation assay (Fig. 3), this dopamine-mediated heterosynaptic LTD may be a common feature for most GABAergic synapses on NAcCo neurons. With this heterosynaptic LTD, the incentive-elicited activation of the NAc can be regulated in a time-contingent manner.

Second, as mentioned above, if the LTD demonstrated here indeed serves as an efficient mechanism to relieve the basal inhibition of NAcCo MSNs upon incentive stimuli such that NAc neurons can engage in the processing of related emotional/motivational information, a conceivable consequence of the disruption of this LTD is that the activity pattern of VTA-to-NAcCo transmission is locked, resulting in compromised flexibility/capability of NAcCo to process incentive stimuli after cocaine withdrawal. This may correspond to several withdrawal symptoms that promote relapse: a rigid emotional state favorable to drug taking, reduced responses to non-drug-related emotional and motivational stimuli, and persistent hypoactivity of NAc (Koob and Le Moal, 2006).

The heterosynaptic LTD described herein suggests a novel role of dopamine as a plasticity trigger, and this unique interaction between dopaminergic and GABAergic signaling within the VTA-to-NAcCo projection may provide a new angle in exploring the structural and physiological basis of the mesocorticolimbic dopamine system and addiction-related neural adaptations.

\section{References}

Atasoy D, Aponte Y, Su HH, Sternson SM (2008) A FLEX switch targets Channelrhodopsin-2 to multiple cell types for imaging and long-range circuit mapping. J Neurosci 28:7025-7030. CrossRef Medline

Brown MT, Tan KR, O'Connor EC, Nikonenko I, Muller D, Luscher C (2012) Ventral tegmental area GABA projections pause accumbal cholinergic interneurons to enhance associative learning. Nature.

Brown TE, Lee BR, Mu P, Ferguson D, Dietz D, Ohnishi YN, Lin Y, Suska A, Ishikawa M, Huang YH, Shen H, Kalivas PW, Sorg BA, Zukin RS, Nestler EJ, Dong Y, Schlüter OM (2011) A Silent Synapse-Based Mechanism for Cocaine-Induced Locomotor Sensitization. J Neurosci 31:8163-8174. CrossRef Medline

Calabresi P, Picconi B, Tozzi A, Di Filippo M (2007) Dopamine-mediated regulation of corticostriatal synaptic plasticity. Trends Neurosci 30:211219. CrossRef Medline

Carr DB, Sesack SR (2000) Projections from the rat prefrontal cortex to the ventral tegmental area: target specificity in the synaptic associations with mesoaccumbens and mesocortical neurons. J Neurosci 20:3864-3873. Medline

Castillo PE, Chiu CQ, Carroll RC (2011) Long-term plasticity at inhibitory synapses. Curr Opin Neurobiol 21:328-338. CrossRef Medline

Chalifoux JR, Carter AG (2011) GABAB receptor modulation of synaptic function. Curr Opin Neurobiol 21:339-344. CrossRef Medline

Chiu CQ, Puente N, Grandes P, Castillo PE (2010) Dopaminergic Modula- 
tion of Endocannabinoid-Mediated Plasticity at GABAergic Synapses in the Prefrontal Cortex. J Neurosci 30:7236-7248. CrossRef Medline

Fiorillo CD, Tobler PN, Schultz W (2003) Discrete coding of reward probability and uncertainty by dopamine neurons. Science 299:1898-1902. CrossRef Medline

Heifets BD, Chevaleyre V, Castillo PE (2008) Interneuron activity controls endocannabinoid-mediated presynaptic plasticity through calcineurin. Proc Natl Acad Sci U S A 105:10250-10255. CrossRef Medline

Huang YH, Ishikawa M, Lee BR, Nakanishi N, Schlüter OM, Dong Y (2011) Searching for presynaptic NMDA receptors in the nucleus accumbens. J Neurosci 31:18453-18463. CrossRef Medline

Ishikawa M, Mu P, Moyer JT, Wolf JA, Quock RM, Davies NM, Hu XT, Schlüter OM, Dong Y (2009) Homeostatic synapse-driven membrane plasticity in nucleus accumbens neurons. J Neurosci 29:5820-5831. CrossRef Medline

Kasanetz F, Deroche-Gamonet V, Berson N, Balado E, Lafourcade M, Manzoni O, Piazza PV (2010) Transition to addiction is associated with a persistent impairment in synaptic plasticity. Science 328:1709-1712. CrossRef Medline

Koob GF, Le Moal M (2006) Neurobiology of addiction. San Diego: Academic.

Lavin A, Nogueira L, Lapish CC, Wightman RM, Phillips PE, Seamans JK (2005) Mesocortical dopamine neurons operate in distinct temporal domains using multimodal signaling. J Neurosci 25:5013-5023. CrossRef Medline

Lindeberg J, Usoskin D, Bengtsson H, Gustafsson A, Kylberg A, Söderström S, Ebendal T (2004) Transgenic expression of Cre recombinase from the tyrosine hydroxylase locus. Genesis 40:67-73. CrossRef Medline

Morishita W, Sastry BR (1996) Postsynaptic mechanisms underlying longterm depression of GABAergic transmission in neurons of the deep cerebellar nuclei. J Neurophysiol 76:59-68. Medline

Mu P, Moyer JT, Ishikawa M, Zhang Y, Panksepp J, Sorg BA, Schlüter OM,
Dong Y (2010) Exposure to cocaine dynamically regulates the intrinsic membrane excitability of nucleus accumbens neurons. J Neurosci 30: 3689-3699. CrossRef Medline

Nair-Roberts RG, Chatelain-Badie SD, Benson E, White-Cooper H, Bolam JP, Ungless MA (2008) Stereological estimates of dopaminergic, GABAergic and glutamatergic neurons in the ventral tegmental area, substantia nigra and retrorubral field in the rat. Neuroscience 152 : 1024-1031. CrossRef Medline

Petreanu L, Mao T, Sternson SM, Svoboda K (2009) The subcellular organization of neocortical excitatory connections. Nature 457:11421145. CrossRef Medline

Steffensen SC, Svingos AL, Pickel VM, Henriksen SJ (1998) Electrophysiological characterization of GABAergic neurons in the ventral tegmental area. J Neurosci 18:8003-8015. Medline

Stuber GD, Hnasko TS, Britt JP, Edwards RH, Bonci A (2010) Dopaminergic terminals in the nucleus accumbens but not the dorsal striatum corelease glutamate. J Neurosci 30:8229-8233. CrossRef Medline

Sulzer D, Joyce MP, Lin L, Geldwert D, Haber SN, Hattori T, Rayport S (1998) Dopamine neurons make glutamatergic synapses in vitro. J Neurosci 18:4588-4602. Medline

Suska A, Lee BR, Huang YH, Dong Y, Schluter OM (2013) Selective presynaptic enhancement of the prefrontal cortex to nucleus accumbens pathway by cocaine. Proc Natl Acad Sci U S A 110:713-718. CrossRef Medline

Taniguchi H, He M, Wu P, Kim S, Paik R, Sugino K, Kvitsani D, Fu Y, Lu J, Lin Y, Miyoshi G, Shima Y, Fishell G, Nelson SB, Huang ZJ (2011) A resource of Cre driver lines for genetic targeting of GABAergic neurons in cerebral cortex. Neuron 71:995-1013. CrossRef Medline

Tritsch NX, Ding JB, Sabatini BL (2012) Dopaminergic neurons inhibit striatal output through noncanonical release of GABA. Nature 490:262-266. CrossRef Medline

Wise RA (1987) The role of reward pathways in the development of drug dependence. Pharmacol Ther 35:227-263. CrossRef Medline 